\title{
Instrumental Configuration of Electromagnetic Thermography and Optical Thermography
}

\author{
Haoran $\mathrm{Li}^{\mathrm{a}}$, Yuming Zhang ${ }^{\mathrm{a}}$, Shunyao $\mathrm{Wu}^{\mathrm{a}}$, Bin Gao ${ }^{\mathrm{a}, 1}$, Guiyun Tian ${ }^{\mathrm{b}}$, Yang Yang ${ }^{\mathrm{c}}$, \\ Yongjie $\mathrm{Yu}^{\mathrm{c}}$ \\ a School of Automation Engineering, University of Electronic Science and Technology \\ of China, China \\ ${ }^{\mathrm{b}}$ School of Engineering, Newcastle University, UK \\ ${ }^{\mathrm{c}}$ Chengdu aircraft industry Co., Ltd.
}

\begin{abstract}
Electromagnetic thermography and optical thermography are both important non-destructive testing (NDT) methods that have been widely used in the fields of modern aerospace, renewable energy, nuclear industry, etc. The excitation modes are crucial whose performances have a decisive effect on the detection results. Previous studies mainly focused on the physics mechanism, applications, and signal processing algorithms. However, the instrument configuration is rarely presented. This paper is to introduces the recently designed excitation sources of electromagnetic thermography and optical thermography detection systems, respectively. These instruments involved L-shaped and Shuttle-shaped sensor structures for electromagnetic thermography and multi-modes excitation for optical thermography. Besides, the topologies and operating principles are shown in detail. Experimental results are carried out to verify the practicability and reliability of the proposed systems.
\end{abstract}

Keywords. Electromagnetic thermography, optical thermography, excitation system, non-destructive testing (NDT)

\section{Introduction}

Thermography instruments and modes have been widely used in the sensing and detection fields as a part of the system, such as laser detection [1], electromagnetic thermography testing [2], ultrasound inspection [3], plasma detection [4] and optical thermography testing [5]. In particular, there are many commercial products in electromagnetic thermography field such as EASYHEAT series produced from Ameritherm, automatic eddy current thermography system designed by Starmans [6], integrated system of eddy current and eddy current thermography developed by Laval University [7] and automatic inspection system developed by Siemens for motor components [8]. In addition, Thermal Wave Imaging (TWI) has developed an optical thermography system for NDT since different excitation modes are integrated. This paper particularly interprets the instrumental configuration for electromagnetic thermography and optical thermography systems to enhance the quality of NDT.

\footnotetext{
1 Corresponding Author: Bin Gao, E-mail: bin_gao@uestc.edu.cn.
} 


\section{Instrument configuration and detection results of the thermography NDT systems}

\subsection{Configuration and detection results of electromagnetic thermography system}

\section{1) Configuration}

The electromagnetic thermography excitation system [9] is composed of an inductive heating device, a synchronizer trigger, a working head and an excitation magnet yoke. Especially, according to different application scenarios, the shape of the magnet yoke can be selected as L-Shaped [10] or Shuttle-shaped [11]. Both of them have good performance in natural micro-crack detection applications.

Figure 1 shows the diagram of the electromagnetic thermography system. When the control device generates a signal to activate the system, high-frequency pulsed current flows through the helix coil and generates an alternating magnetic field. The ferrite core collects the magnetic flux and the flux is transmitted to the sample between the two poles of the core through a magnetic circuit. These include ferrite core, the air gap and the sample. If a defect exists in the conductive material, the distribution of the magnetic field, the eddy current intensity, and the process of thermal diffusion will be affected. Consequently, the transient time-spatial characteristics of radiative heat transfer modes over the plate surface can be captured by an infrared camera.

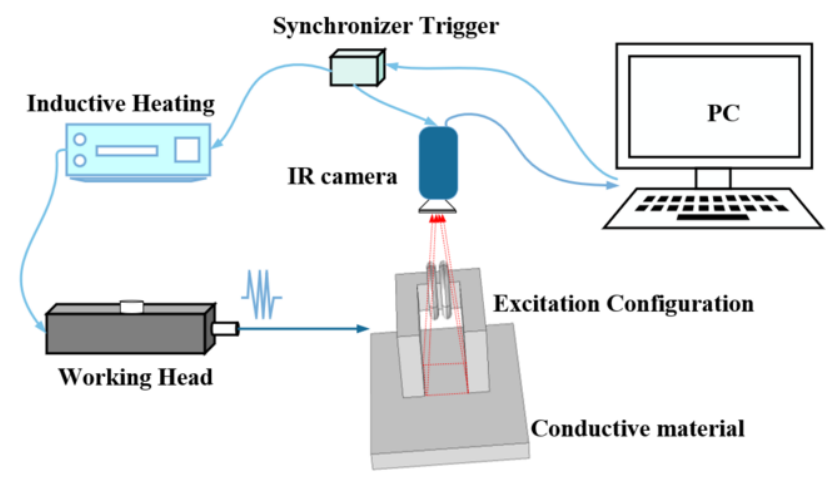

Figure 1. Diagram of the electromagnetic thermography system

Figure 2 presents the experimental system. As shown in Figure 2, the inductive heating device can be further divided into a full-bridge LC resonant circuit, an FPGA control board, a high-power DC power supply, and an auxiliary power supply. The topology of the full-bridge LC resonant circuit is shown in Figure 3. $S_{1}-S_{4}$ are four power MOSFETs, $V_{S}$ is the voltage provided by DC power supply and its value is adjustable from $0-60 \mathrm{~V}$. 


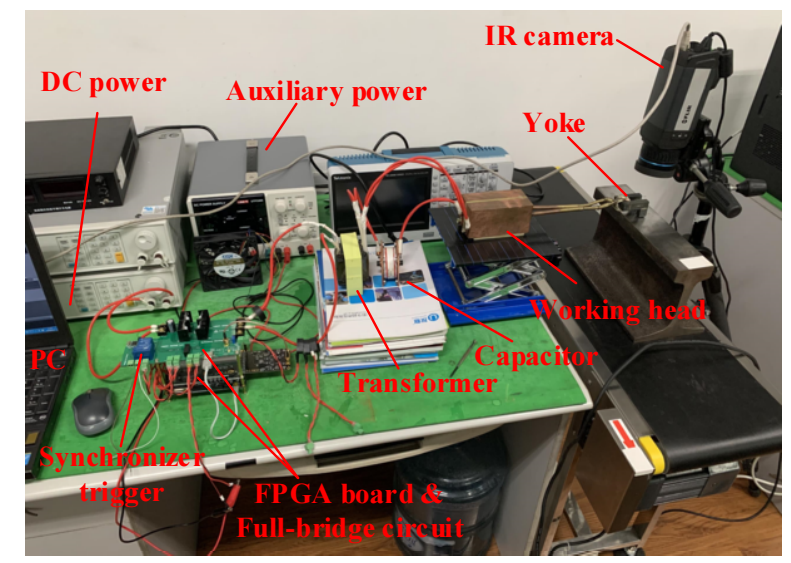

Figure 2. Experimental system

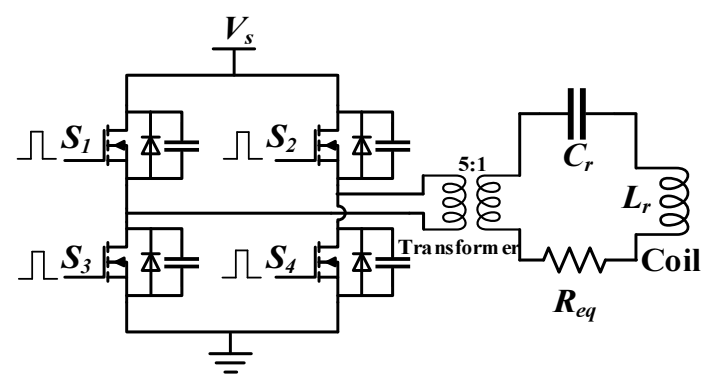

Figure 3. The topology of the full-bridge LC resonant circuit

In addition, $R_{e q}$ denotes the equivalent resistance of the circuit, it is typically ranged from several dozen to several hundred $\mathrm{m} \Omega$. $C_{r}$ denotes the resonant capacitor and the value is $0.4 \mu \mathrm{F}$. $L_{r}$ denotes the resonant inductance and the value is $1 \mu \mathrm{H}$. The turns ratio of the transformer is $5: 1$. When the circuit works at the resonant state, the current flowing through the excitation coil reaches a peak value, and the magnetic field generated by the coil is the strongest. The resonant frequency of the series resonant circuit $f_{r}$ can be described as:

$$
f_{r}=\frac{1}{2 \pi \sqrt{L_{r} C_{r}}}
$$

The working logic of the excitation system can be summarized as follows: Firstly, the FPGA is programmed as the drive frequency sweep from $300 \mathrm{kHz}-50 \mathrm{kHz}$ and acquire the secondary current of the transformer during this process. Secondly, if this current value is greater than a set threshold, it means that the frequency is close to the resonant frequency and mark this value. Finally, the FPGA will stop sweeping and output at the marked frequency. Thus, the system will find the resonant frequency automatically and generate the maximum output power which is important to inspection. At this working state, the maximum peak-to-peak value of output current is $\pm 180 \mathrm{~A}$ which is suitable for electromagnetic thermography testing. 


\section{2) Detection results}

The verification experiments are performed by using the proposed excitation configuration model with L-shaped and Shuttle-shaped yoke respectively to detect natural microcracks on different samples. The excitation current is set to $180 \mathrm{~A}$ and frequency is matched on $220 \mathrm{kHz}$. Thermal images are captured by the infrared camera as shown in Figure 4 and Figure 5, respectively. It can be seen that the multiple natural cracks in complex geometry can be visualized effectively.
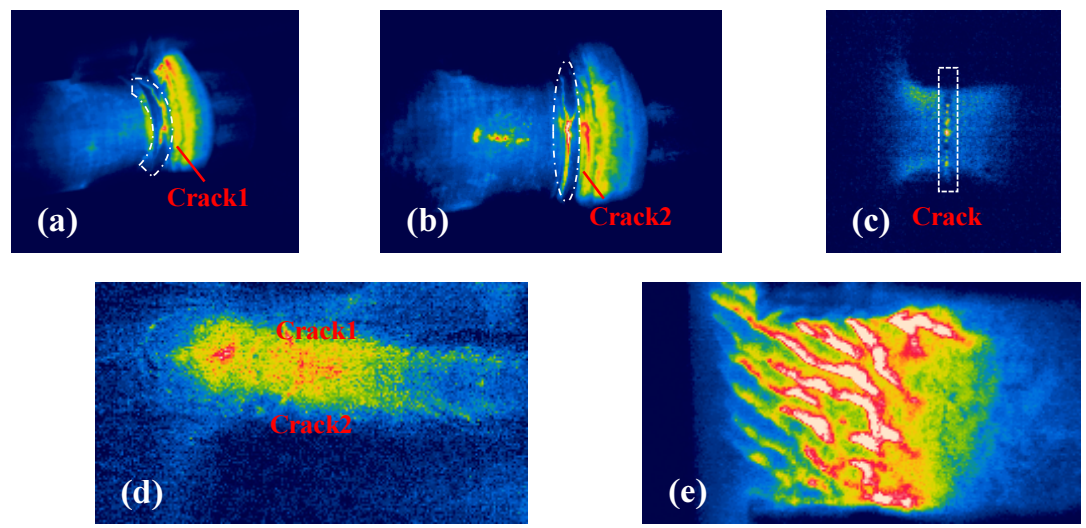

Figure 4. Detection results of (a)(b) screw (c) stainless steel plate (d) weld crack and (e) RCFs by using L-shaped yoke
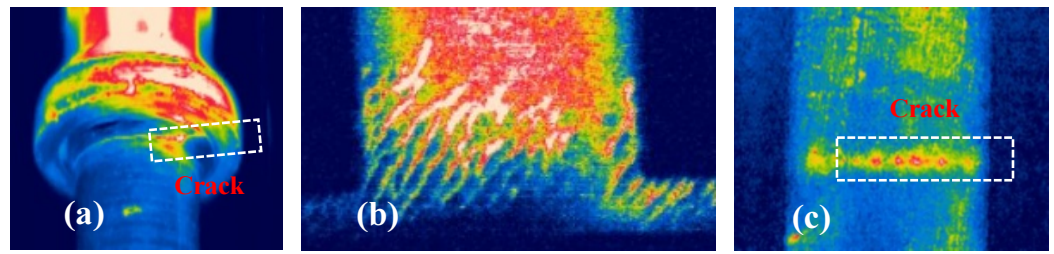

Figure 5. Detection results of (a) screw (b) RCFs (c) stainless steel plate by using Shuttle-shaped yoke

\subsection{Configuration and detection results of potable optical thermography system}

\section{1) Configuration}

Carbon fiber reinforced polymer (CFRP) has become an indispensable structural material in the aircraft manufacturing industry. However, quality issues in CFRP may be caused by cyclic stress, impact damages and any other anomaly buried within the composite structure. Defects associated with composite materials will lead to a significant reduction of the load capacity and degradation of mechanical properties. Therefore, it is necessary to conduct a reliable nondestructive testing analysis to guarantee safety.

To meet this demand, Portable Optical Pulsed Thermography (POPT) system is designed for defect detection in carbon fiber reinforced composite in reflection mode, using halogen lamps as an excitation source. As shown in Figure 6(a), it includes an excitation source and a CPU, a display screen, six halogen lamps with a power of $900 \mathrm{~W}$, an IR camera, and a grip. The temperature sensitivity is $0.06^{\circ} \mathrm{C}$, and the maximum resolution is $288 \times 384$. Figure 6 (b) illustrates the new configuration of human-computer interaction interface which is developed using $\mathrm{C \#}$. 


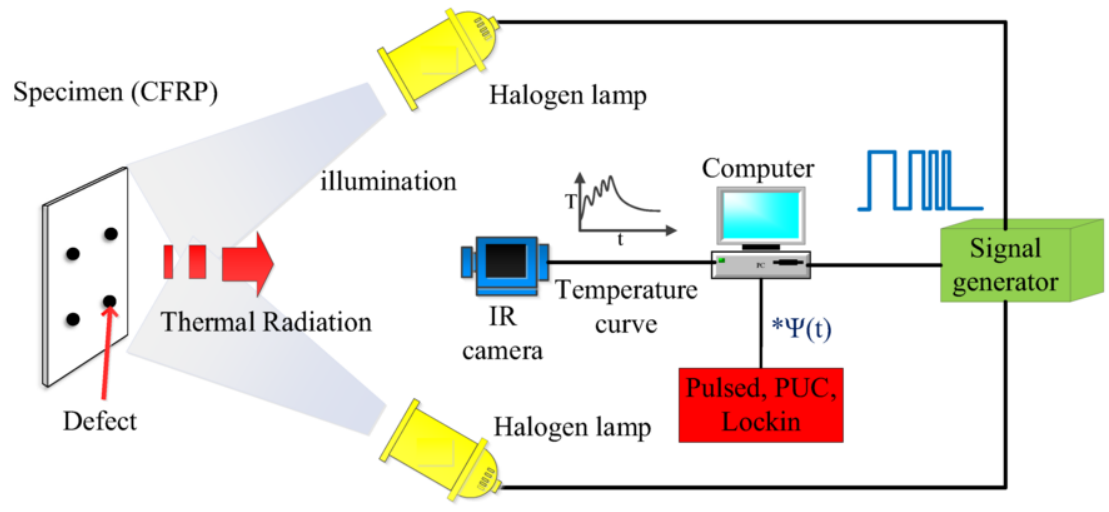

(a) Diagram of the POPT system
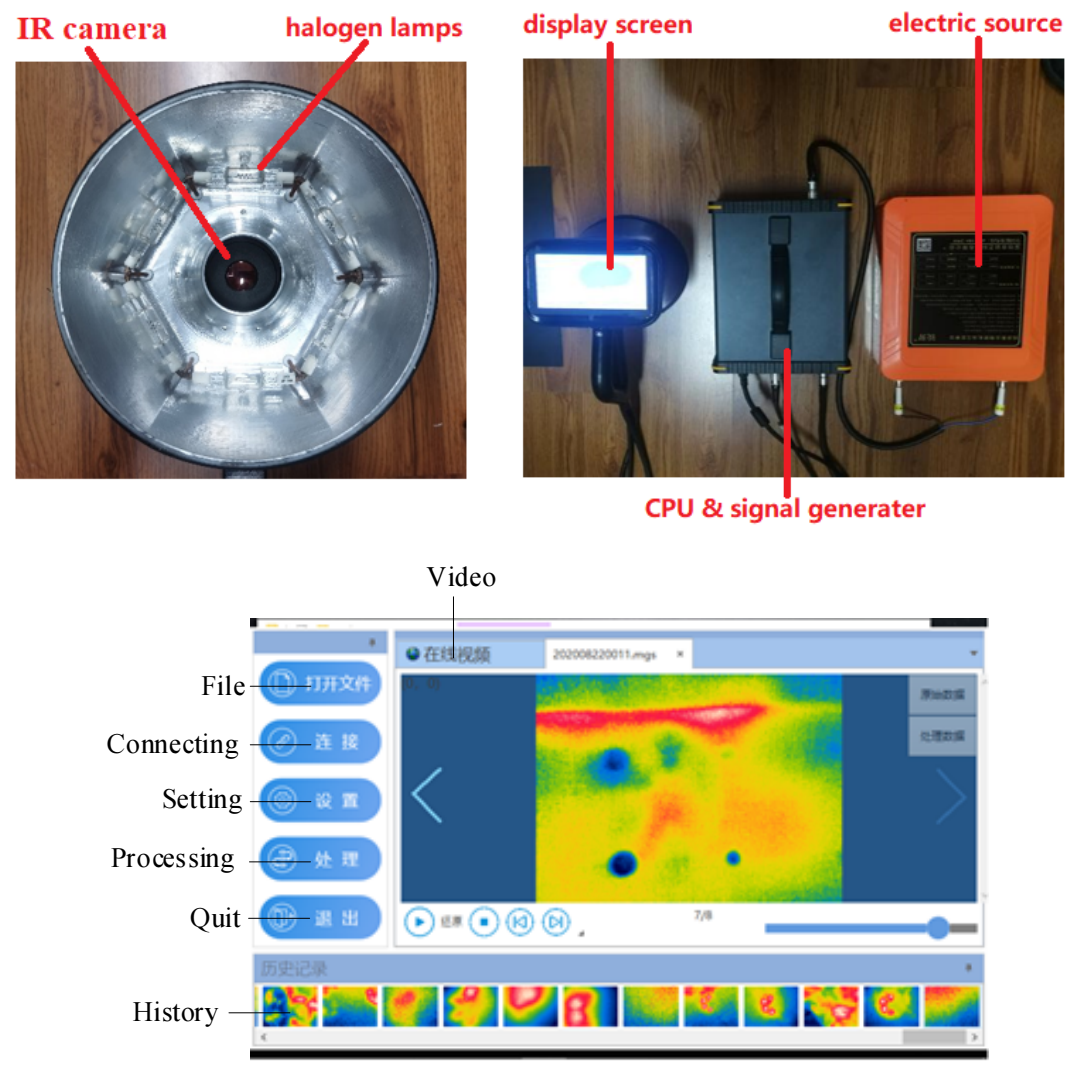

(b) The physical picture of POPT system

Figure 6. The components and structure of the POPT system

After setting the parameters on the touch screen, the excitation source sends signals to the halogen lamps, and the IR camera records the heating and cooling stages of the specimen, which are stored in the form of video and processed by the tensor decomposition [12] algorithms and deep learning methods. 


\section{2) Detection results}

The detection results of carbon fiber board with buried defects are shown in Figure 7, which provides the evidence of the reliability of the POPT system. Large curvature surface can be captured and detected. More quantitative analysis and comparison can be found in [13]. It should be noted that in these experiments long pulse excitation is chosen and the heating time is set to $3 \mathrm{~s}$. Furthermore, the excitation power is $900 \mathrm{~W}$ and the cooling time is $3-4 \mathrm{~s}$.
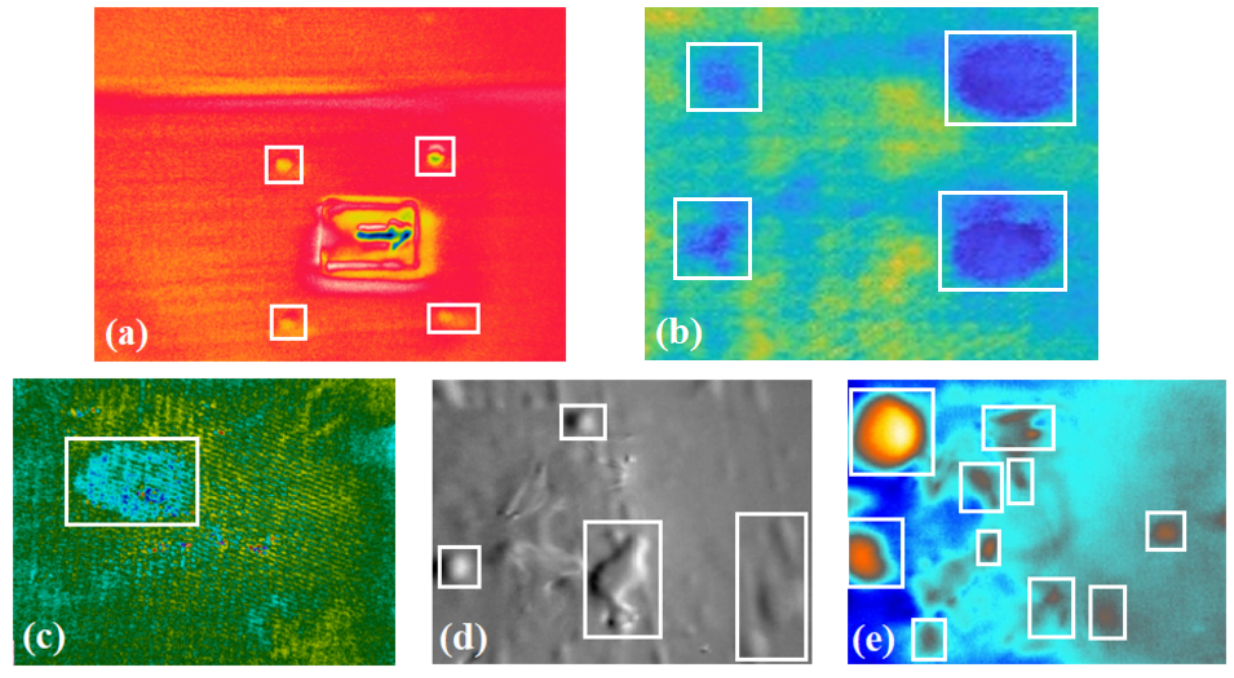

Figure 7. Detection results of artificial defects (a) (b) delamination (b) bubble (c) and debonding (d)\&(e)

\section{Conclusion}

This paper presents the instrumental configuration of two modes of thermography NDT system. The natural micro-cracks on different samples can be detected obviously through the electromagnetic thermography excitation system. In addition, the POPT system can effectively separate the defect information from thermal diagrams by using the tensor decomposition method [14]. Both of the two systems have the advantage of small volume which means they have the potential to be portable and more conducive to non-laboratory condition testing. However, it is still a challenge for these systems to distinguish all the defects especially in some irregular shape components.

\section{Acknowledgement}

This work was supported by Defense Industrial Technology Development Program (Grant No. JSZL2019205C003), National Natural Science Foundation of China (No. 61971093, No. 61527803, No. 61960206010). The work was supported by Science and Technology Department of Sichuan, China (Grant No.2019YJ0208, Grant No.2018JY0655, Grant No. 2018GZ0047) and Fundamental Research Funds for the Central Universities (Grant No. ZYGX2019J067). 


\section{References}

[1] Asher S A. "Some Important Considerations in the Selection of a Tunable UV Laser Excitation Source, " Applied Spectroscopy, vol. 38(2), pp. 276-278, Mar 1984.

[2] Sfarra, Stefano, et al. "Quantitative Infrared Thermography (IRT) and Holographic Interferometry (HI): Nondestructive Testing (NDT) for Defects Detection in the Silicate Ceramics Industry." Advances in Science \& Technology, vol. 68, pp. 102-107, Oct 2010.

[3] Poelzleitner, Wolfgang. "Ultrasound lock-in thermography: feasibilities and limitations," Proc Spie, vol. 3827, pp. 10-15, Sep 1999.

[4] Abdallah M H, Coulombe S, Mermet J M, et al. "An assessment of an atmospheric pressure helium microwave plasma produced by a surfatron as an excitation source in atomic emission spectroscopy," Spectrochimica Acta Part B Atomic Spectroscopy, vol. 37(7), pp. 583-592. 1982.

[5] Y. Zhu et al., "Nondestructive Testing for Multi-Layer Metal-Metal Bonded Structure by Using Inductive Lock-In Thermograhy," IEEE Sensors Journal, vol. 17, no. 20, pp. 6716-6723, 15 Oct.15, 2017, doi: 10.1109/JSEN.2017.2741581.

[6] Staeman S, Matz V. "Automated System for Crack Detection Using Infrared Thermographic Testing." In 17th World Conference on Nondestructive Testing. Shanghai: 2008.

[7] Grenier M, et al. "Development of a hybrid non-destructive inspection system combining induction thermography and eddy current techniques." In 10th international conference on quantitative infrared thermography. Quebec: 2010.

[8] Goldammer M, et al. "Automated Induction Thermography of Generator Components." AIP Conference Proceedings, 1211(2010):451-457.

[9] H. Li, et al., "Multiphysics Structured Eddy Current and Thermography Defects Diagnostics System in Moving Mode," IEEE Transactions on Industrial Informatics, doi: 10.1109/TII.2020.2997836.

[10] Z. Liu, B. Gao and G. Y. Tian, "Natural Crack Diagnosis System Based on Novel L-Shaped Electromagnetic Sensing Thermography," IEEE Transactions on Industrial Electronics, vol. 67, no. 11, pp. 9703-9714, Nov. 2020, doi: 10.1109/TIE.2019.2952782.

[11] X. Li, B. Gao, Z. Liu and G. Y. Tian, "Microcracks Detection Based on Shuttle-shaped Electromagnetic Thermography," IEEE Sensors Journal, doi: 10.1109/JSEN.2020.3001305.

[12] L. Liu, et al, "Structured Iterative Alternating Sparse Matrix Decomposition for Thermal Imaging Diagnostic System," Infrared Physics and Technology, vol. 107, no. 2020, pp. 1-14, Apr 2020.

[13] Z. Wang, J. Zhu, G. Y. Tian and F. Ciampa, "Comparative analysis of eddy current pulsed thermography and long pulse thermography for damage detection in metals and composites," NDT \& E International vol. 107, 102155, 2019.

[14] J. Ahmed, B. Gao, W. L. Woo and Y. Zhu, "Ensemble Joint Sparse Low Rank Matrix Decomposition for Thermography Diagnosis System," IEEE Transactions on Industrial Electronics, doi: 10.1109/TIE.2020.2975484. 